\title{
DYNAMICS OF INTRACELLULAR CALCIUM AND ITS POSSIBLE RELATIONSHIP TO PHASIC TRANSMITTER RELEASE AND FACILITATION AT THE FROG NEUROMUSCULAR JUNCTION ${ }^{1}$
}

\author{
NORMAN STOCKBRIDGE ${ }^{2}$ AND JOHN W. MOORE
}

Department of Physiology, Duke University, Durham North Carolina 27710

Received September 8, 1982; Revised August 29, 1983; Accepted October 19, 1983

\begin{abstract}
We have developed a mechanistic model for intracellular influx, diffusion, and efflux of calcium, and we compare its predictions to the dynamics of transmitter release at the frog's motor nerve terminal.

The model includes a square wave influx of calcium, a linear or saturable pump for the efflux of calcium, and slow diffusion of calcium within the terminal due to rapid equilibrium binding of the major portion of the influx to fixed, nonsaturable sites in the cytoplasm. Transmitter release is taken as proportional to the fourth power of the calcium concentration in a region within $100 \AA$ of the surface membrane. The model predicts phasic release of transmitter with a time course similar to that of the endplate current-it turns on with a lag, rises rapidly to a peak, and then declines more slowly. It also predicts facilitation, the increased transmitter release in response to a second stimulus for many milliseconds after the initial transmitter release has ceased. This facilitation is similar to that reported at the frog neuromuscular junction in its initial amplitude and time course.
\end{abstract}

Although the release of transmitter at the frog neuromuscular junction has been extensively studied, no mechanism has been offered to account simultaneously for phasic release of transmitter and its facilitation. Katz and Miledi (1976b) showed that external calcium was necessary at the time of the first nerve impulse and suggested that calcium entered the terminal and, by binding to some receptor site at the presynaptic terminal, released transmitter. The "residual calcium" hypothesis for facilitation evolved from their suggestion (Katz and Miledi, 1965b) that a residuum of "active calcium" remains within the terminal following an action potential for several milliseconds and is able to enhance the amount of transmitter released by succeeding stimuli.

Most models proposed for facilitation do not include any mechanisms but are merely mathematical descriptions of the envelope of peak postsynaptic potentials. Most avoid the crucial question: If transmitter release is proportional to the internal calcium concentration how can it be reduced rapidly for phasic release and yet be high enough to show facilitation for many milliseconds? Katz and Miledi (1968) raised this question and con-

\footnotetext{
${ }^{1}$ We are grateful for the support of this work by National Institutes of Health Grants NS 03437, NS11613, and GM07046 to J. W. M. This study comprised part of a doctoral dissertation for N. S.

${ }^{2}$ Present address: Department of Physiology, New York Medical College, Valhalla, NY 10595.

${ }^{3}$ To whom correspondence should be addressed.
}

cluded that a full answer was not provided by the assumption that transmitter release is proportional to the fourth power of the calcium concentration.

Rather than try to fit another ad hoc kinetic scheme to experimental observations, we have pursued a different tack, that of using computer simulations of known mechanisms to provide insight into the functioning of a phasic synapse. We have constructed rather simple models of the mechanisms for the control of calcium which are known to be present at nerve terminals: influx, efflux, intracellular binding, and diffusion. Our limited goal is to account for only two characteristics of transmitter release: (1) the rapid transient rise and decay of phasic release and (2) the dynamics of facilitation.

There is no direct measure of transmitter release on a millisecond time scale. The endplate potential amplitude is roughly proportional to the time integral of free transmitter in the synaptic cleft. The endplate current gives a more realistic (but still inaccurate) reflection of the transmitter in the cleft, and we use this pattern as the best available measure of release. Phasic release (endplate current) at the neuromuscular junction of the frog begins after a delay of $0.5 \mathrm{msec}$ (at room temperature) following the arrival of a nerve impulse. The rising phase then lasts $0.5 \mathrm{msec}$, and the falling phase has a time constant of about $1 \mathrm{msec}$ (Takeuchi and Takeuchi, 1959; Magleby and Stevens 1972).

The second characteristic of transmitter release for 
which a model must account is that following single stimuli or trains of stimuli, there is a time during which succeeding stimuli will evoke the release of increased amounts of transmitter. This characteristic has been described as four processes, distinguished by their time constants for decay. The slower pair are referred to as augmentation and potentiation and, because of their low amplitudes, become evident only following trains. Because augmentation and potentiation arise and decay over periods of seconds, times long compared with the processes in this model, they will not be dealt with in the current work. The faster pair of processes, referred to together as facilitation, are evident following single stimuli. As described by Mallart and Martin (1967), facilitation appears at its peak immediately after a conditioning pulse and decays with time constants of about 35 to 50 msec and 200 to $300 \mathrm{msec}$.

Our first model incorporated the conventional assumption that transmitter release was proportional to the internal free calcium, which in turn was assumed to be uniform throughout the terminal. The model included pulsative entry of calcium with each impulse, a linear pump, and two types of binding (a large number of low affinity sites and a small number of high affinity sites). This model showed (1) rapid decay of internal calcium following each phasic entry and (2) a build-up of internal free calcium with successive calcium entries in a closely spaced train. However, instead of leveling off at a higher value, the calcium peaks increased without limit as the binding sites became saturated. Even with the more realistic assumption (see arguments below) that transmitter release was proportional to the fourth power of internal calcium, this problem persisted.

Therefore, it was necessary to proceed to a more realistic model which included the spatial distribution of internal calcium. This model incorporates the Blaustein and Hodgkin (1969) assumption that the diffusion coefficient for intracellular calcium is reduced dramatically by the presence of a large number of binding sites.

\section{Model}

Calcium's role in transmitter release. Several avenues of research have examined different aspects of the role of internal calcium in transmitter release.

Some studies indicate that internal calcium results in release. (1) Miledi (1973) showed that transmitter release could be stimulated at the squid giant synapse by intracellular iontophoresis of calcium but not other divalent cations. (2) Rahamimoff et al. (1978b) exposed frog neuromuscular junctions to calcium-containing liposomes and found that they increased release while lipsomes with other ions do not. (3) Rahamimoff et al. (1978a) placed frog neuromuscular junctions in low calcium Ringer and found that evoked transmitter release steadily decreased with successive stimuli-interpreted as indicating a reversal of the calcium concentration gradient with the consequent loss of calcium to the bath with stimulation i.e., "negative facilitation."

The quantitatve relationship between external calcium and transmitter release has been shown (Dodge and Rahamimoff, 1967; Andreu and Barrett, 1980) that transmitter release was proportional to the fourth power of the bath concentration of calcium.
The kinetics of release can be described as a first-order reaction raised to the third or fourth power. Katz and Miledi (1965a) studied kinetics of transmitter release by analysis of fluctuations in latency (which is mostly presynaptic). Barrett and Stevens (1972) extended these findings by developing an expression for the release probability function derived from the probability distribution of the first quantal latencies. As did Katz and Miledi, they found that the early release probability rose quickly to a peak and declined more slowly with a single time constant of about $0.5 \mathrm{msec}$ at $11^{\circ} \mathrm{C}$. In addition, Barrett and Stevens found a slower decay which could be fit by the sum of two exponentials having time constants of 15 to $20 \mathrm{msec}$ and $230 \mathrm{msec}\left(\right.$ at $\left.1^{\circ} \mathrm{C}\right)$. In addition, there was some evidence for a process with an even longer time constant. They hypothesize that the early decline is due to calcium buffering and that the later process is due to slower uptake, as by mitochondria, or to extrusion. Furthermore, they fitted the early release of facilitation of the residual release probability with a third or fourth power of a linear process like that of Dodge and Rahamimoff (1967).

'To summarize, (1) direct injection of calcium into the terminal results in transmitter release, (2) external calcium ions are necessary to support transmitter release by nerve impulses, (3) calcium entry into the terminal with each impulse has been demonstrated, (4) there is a fourth power relationship between external calcium and transmitter release.

Model components. The model for calcium metabolism we examined is illustrated diagrammatically in Figure 1 and consists of (1) calcium influx, (2) slow diffusion of calcium due to rapid equilibrium binding of most calcium ions to a uniformly distributed and unsaturable buffer (implying relatively low calcium concentrations), and (3) calcium efflux, either linearly related to the internal calcium concentration near the cell membrane, or some saturable function of the calcium concentration.

In the model, we take the fourth power of the internal calcium concentration as the release function, the time course of which is compared with the time course of transmitter release as measured by endplate currents. We have made this choice for two reasons. First, under no conditions have we found similarity between the kinetics of transmitter release and the first power of the internal calcium concentration. Second, we think that the increase of calcium concentration just under the excitable membrane following an impulse should be proportional to the external concentration. Because the gradient for calcium across the cell membrane is so high, we argue that the inward flux is largely determined by diffusion, and that the fourth power relation observed for external calcium and transmitter release probably reflects a similar strong dependence of release on internal calcium.

Calcium influx. Because Katz and Miledi (1967a) showed that, for transmitter to be released, external calcium was necessary at the time of arrival of the nerve impulse, voltage-dependent calcium channels probably exist at the frog's nerve terminal. At the squid giant synapse, influx of calcium into the presynaptic terminal has been demonstrated (by arsenazo III absorption changes) following action potentials (Charlton et al., 


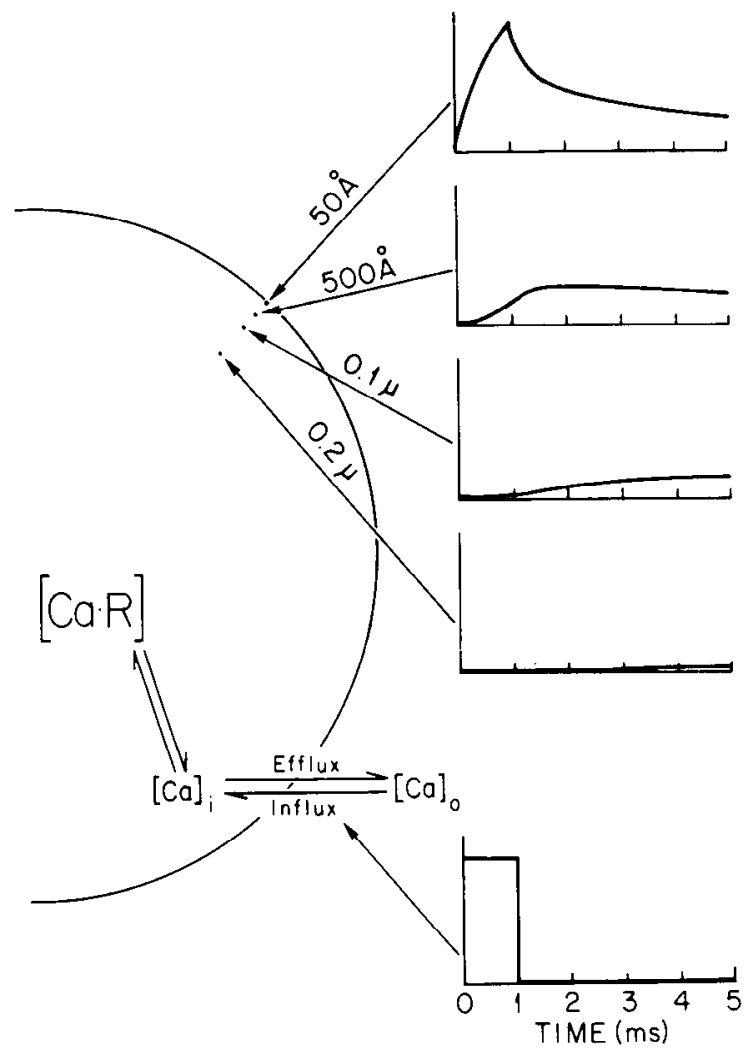

Figure 1. Diagram of the model of the various mechanisms which affect free intracellular calcium concentration on a millisecond time scale. The components are calcium influx (by voltage-dependent calcium channels), efflux (by an energydependent pump), and, within the terminal, diffusion limited by the rapid binding of a large fraction of the total intracellular calcium to cytoplasmic sites. In response to the calcium influx pattern shown in the lower right diagram, the rise and fall of the free calcium concentration are shown at various distances from the cell surface membrane. Note the large transient increase in free calcium just under the membrane.

1982) and under voltage clamp depolarizations (Llinas et al., 1981a).

Calcium influx through ion specific channels is modeled as square pulse having an amplitude of $1000 \mathrm{pmol} /$ $\mathrm{cm}^{2}-\mathrm{sec}$ and a duration of $1 \mathrm{msec}$. A more realistic shape close to that measured by Llinas et al. (1981a) was occasionally used but seemed to have little effect on the parameters which we were studying, as described under "Results."

Calcium binding and diffusion. Axoplasm is the source of a number of components which bind calcium and would be expected to result in slowing the rate of diffusion of intracellular calcium. Baker (1976) described mitochondrial and nonmitochondrial components of high affinity ( 0.3 to $0.5 \mu \mathrm{M})$ and moderate capacity (20 to $40 \mu \mathrm{M})$.

A slow rate of calcium diffusion in cytoplasm (compared to the rate in free solution of $6 \times 10^{-6} \mathrm{~cm}^{2}$-sec) has been measured. Hodgkin and Keynes (1957) found the radial diffusion constant to be $6 \times 10^{-7} \mathrm{~cm}^{2}$-sec using intracellular loads of 3.6 to $18.8 \mathrm{~mm}$. Blaustein and Hodgkin (1969) estimated the rate at $3 \times 10^{-7} \mathrm{~cm}^{2}$-sec using loads of 1 to $3 \mathrm{~mm}$.
Since these loads are all large compared to physiological loads and since the measured diffusion constant decreased in the lesser load, one should regard the value as an upper limit.

Pump. The calcium which enters the cell must be eventually extruded. The types of mechanism by which this is accomplished-in red cells, liver, snail neurons, and squid axon-are (1) sodium-calcium exchange (Baker et al., 1970; Baker, 1972), and (2) an energydependent and sodium-independent pump (DiPolo and Beauge, 1979).

Blaustein (1976) has described extrusion in the squid giant synapse as a saturable process with a maximal rate of $1.7 \mathrm{pmol} / \mathrm{cm}^{2}$-sec, half-maximal at a calcium concentration of $8 \mu \mathrm{M}$. At low calcium concentrations, the relationship is nearly linear with a slope of $1.4 \times 10^{-4}$ $\mathrm{cm} / \mathrm{sec}$. Mullins (1976) reports a linear relation in low calcium with a slope of $10^{-3} \mathrm{~cm} / \mathrm{sec}$. Our simulations include this range of pumping rates and extend it another 10 -fold.

The measurement of the rate constant for extrusion from the efflux depends upon knowledge of the calcium concentration near the membrane since that is the calcium that the extrusion mechanism sees. As calcium is extruded, if there arises a diffusion gradient from the interior of the cell toward the periphery, then an erroneous estimation of the calcium concentration near the membrane will lead to erroneously low values for the calcium pump rate constant. The extent to which this may occur in the nerve terminal is discussed.

Factors omitted. While the role of various intracellular organelles in the control of intracellular calcium has been the subject of a number of investigations, the case remains to be made whether such organelles as mitochondria and smooth endoplasmic reticulum participate in the rapid time course of processes such as transmitter release. Anticipating our calculation that diffusion is sufficiently slow so that the calcium modulating rapid events, such as transmitter release, never encounters such organelles, their contribution to calcium homeostasis will be excluded from the model we present; failure of the model in specific areas may then point to the role filled by such organelles.

No attempt is made here to force transmitter release into discretc quanta. Nor was there explicit inclusion of (1) diffusion of transmitter across the synaptic cleft, (2) the possible generation of concentration gradients in the synaptic cleft. ${ }^{4}$ or (3) the reaction with postsynaptic receptors and subsequent opening of channels in the sarcolemma.

These simplifications seemed reasonable because these steps contribute little to the time course of the endplate response (Katz and Miledi, 1965a).

\footnotetext{
${ }^{4}$ Although less important than gradients within the terminal, simple estimates indicate that it is worthy of consideration in a more complete and realistic model. If the synaptic cleft is $200 \AA$ wide and the extracellular calcium concentration is $1 \mathrm{mM}$, the influx of $1 \mathrm{pmol} / \mathrm{cm}$ into the terminal would result in halving the calcium concentration in the cleft, neglecting diffusion of calcium from regions of the cleft adjacent to active zones or from the bulk solution or that extruded by the muscle and terminal.
} 


\section{Methods}

The nerve terminal was taken to be a $1-\mu \mathrm{m}$ cylinder in which circumferential symmetry and uniform properties in the axial direction were assumed. In order to study the effect of slow diffusion without the interference and added complexity of the time course of calcium influx, influx into the outer shell of the cylindrical cell was modelled as a square wave current of 1-msec duration.

Literature measures of efflux having the limitations described under "Results" and "Discussion," we chose to incorporate a linear pump in which efflux is directly proportional to the calcium concentration in the outermost shell of cytoplasm in the cell. Such efflux can be described by:

$$
J_{e}=k_{e}[\mathrm{Ca}]_{x=0}
$$

where $J_{e}$ (moles per square centimeter per second) is the efflux, $k_{e}$ (centimeters per second) is the flux rate constant and $[\mathrm{Ca}]_{x=0}$ is the calcium concentration of the outermost compartment.

Calcium was assumed to enter the cell from an inexhaustable external medium. The diffusion of calcium within the cell is given by (Crank, 1956):

$$
\frac{\partial \mathrm{C} \mathbf{a}_{x}{ }^{t}}{\partial t}=\frac{D_{f}}{\beta+1} \nabla^{2} \mathrm{Ca}_{x}{ }^{t}
$$

where $\mathrm{Ca}_{x}{ }^{t}$ is the calcium concentration (molar) at a distance $x$ (centimeters) in the radial direction from the membrane at a time $t$ (seconds). $D_{f}$ is the free diffusion
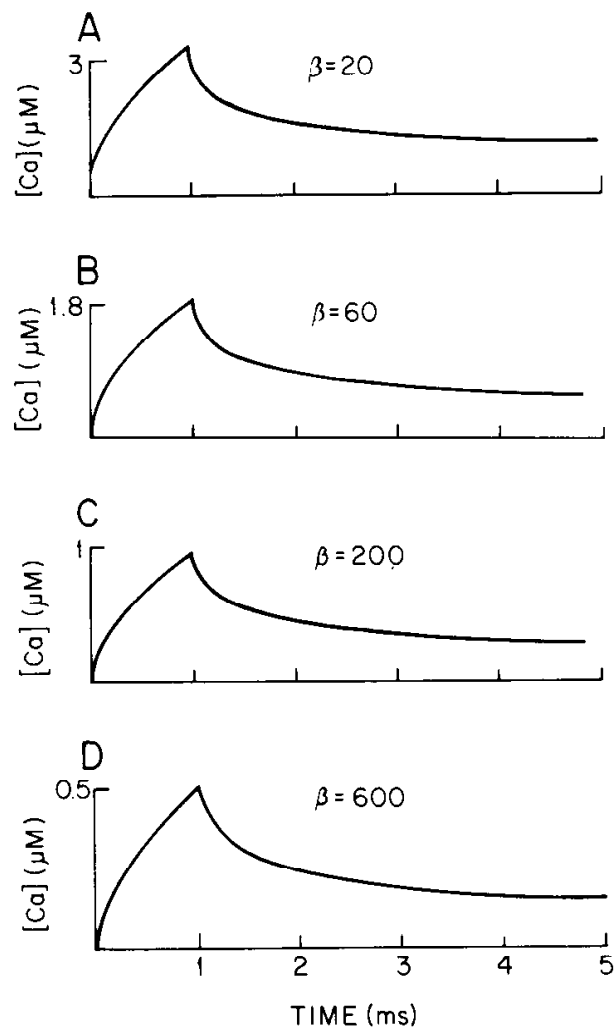

constant for calcium (square centimeters per second). $\beta$ is the ratio of bound to free calcium, and $\nabla^{2}$ is the Laplacian operator.

Rapid equilibrium was assumed to occur between calcium and its binding sites in the cytoplasm. Such binding sites are assumed to be uniformly distributed within the terminal, and their concentration is large compared to the calcium concentration encountered. Thus $\beta$ is independent of both the calcium concentration and time.

For a cylindrical coordinate system with only a radial gradient, we have:

$$
\frac{\partial \mathrm{Ca}_{x}{ }^{t}}{\partial t}=D \frac{1}{x} \frac{\partial}{\partial x}\left(x \frac{\partial \mathrm{Ca}_{x}{ }^{t}}{\partial x}\right)
$$

where $D=D_{f} / \beta+1$. This equation was cast into a finite difference approximation for the partial derivatives. In turn, this was simplified into a tridiagonal matrix and solved by an efficient set of subroutines for which we are grateful to Dr. Michael Hines. The time domain is divided into discrete intervals of duration $t=10 \mu \mathrm{sec}$, and the interior of the cell is divided into discrete annular shells of $100 \AA$ thickness.

\section{Results}

Diffusion. We consider first the effect of radial diffusion alone (with no calcium extrusion mechanism) in a cylindrical cell in which the resting free calcium concentration is $10^{-8} \mathrm{M}$, investigating the effect of $\beta$ ranging from 20 to 600 . Figure 2 illustrates the time course (left side) and the spatial distribution (right side) of the
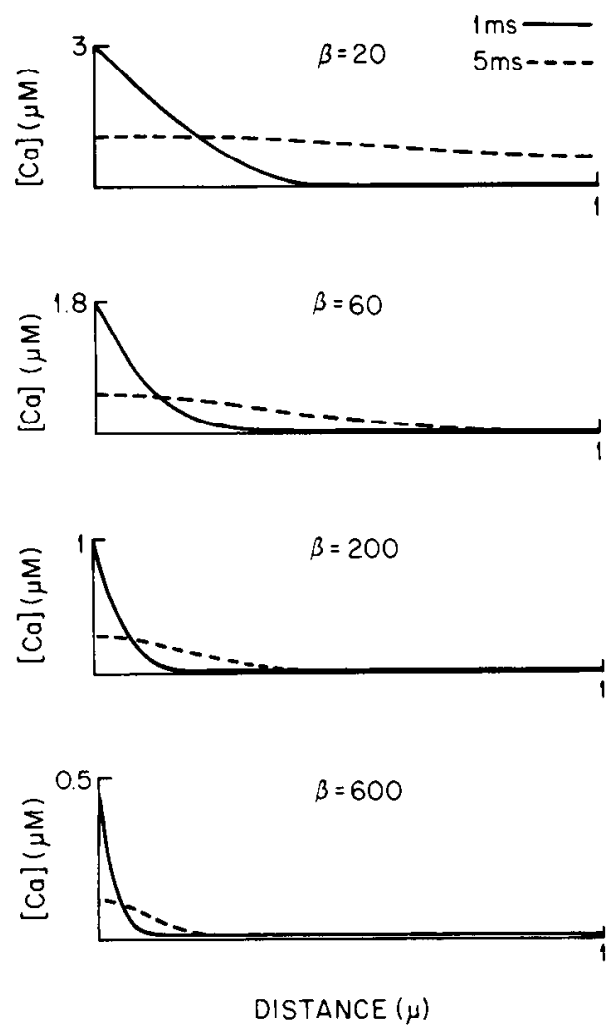

Figure 2. The result of influx (but no efflux) and binding on intracellular calcium transients. $A, B, C$, and $D$ correspond to $\beta \mathrm{S}$ of $20,60,200$, and 600 , respectively. The left side of each part shows the time course of the calcium transient in the outer $100 \AA$ of the model cell. On the right is shown the spatial gradient from the outer margin to the center of the model cell at the end of the stimulus $(1 \mathrm{msec})$ and at $5 \mathrm{msec}$. In each case the total calcium influx was a 1 -msec square wave of $1 \mathrm{pmol} / \mathrm{cm}^{2}$. 
intracellular calcium concentration during and following a $1-\mathrm{msec}$ square wave giving an influx of $1 \mathrm{pmol} / \mathrm{cm}^{2}$. In each case, the plot was normalized to the peak calcium concentration; the largest peak concentration of free calcium occurs with the highest effective diffusion rate. For $\beta=20,60,200$, and 600 , respectively, the free calcium peak concentrations reached at the end of the stimulus are about 3.0, 1.8, 1.0, and $0.5 \mu \mathrm{M}$.

As the number of binding sites increases, the peak concentration of free calcium declines and the diffusion slows, causing a localization of calcium near the membrane. This facilitates removal of calcium by the pump, as can be seen in the spatial distribution plots below. Note particularly that, at $5 \mathrm{msec}$, the calcium concentration has not even begun to rise at a distance of $0.5 \mu \mathrm{m}$ from the surface, if $\beta$ is above 20 .

Diffusion and pump. The effect of the addition of a pump is shown in Figure 3, where $\beta$ was held constant at a value of 50 , a value thought to be reasonable on the basis of considerations raised under "Model." A linear pump with a rate constant of $10^{-4} \mathrm{~cm} / \mathrm{sec}$ (Blaustein, 1976) has negligible effects on this time scale, but a 10fold increase in the pump rate to $10^{-3} \mathrm{~cm} / \mathrm{sec}$ (Mullins, 1976) causes a small change in the calcium distribution (Fig. 3B). The peak calcium concentration near the membrane is reduced slightly, and there is an increase in the rate of decline. A further 10-fold increase in the pump rate (to $10^{-2} \mathrm{~cm} / \mathrm{sec}$, Fig. $3 C$ ) has a more dramatic effect; the peak calcium concentration near the membrane is reduced about one-third; and the decay phase is markedly accelerated.

The spatial distribution is shown again on the right for each case. Shortly following the termination of the influx, the $10^{-2} \mathrm{~cm} / \mathrm{sec}$ pump pulls down the calcium concentration near the membrane quickly enough to establish a reversal of the gradient for diffusion. Further increases in the rate of extrusion have a diminishing effect because the concentration of calcium at the mem-
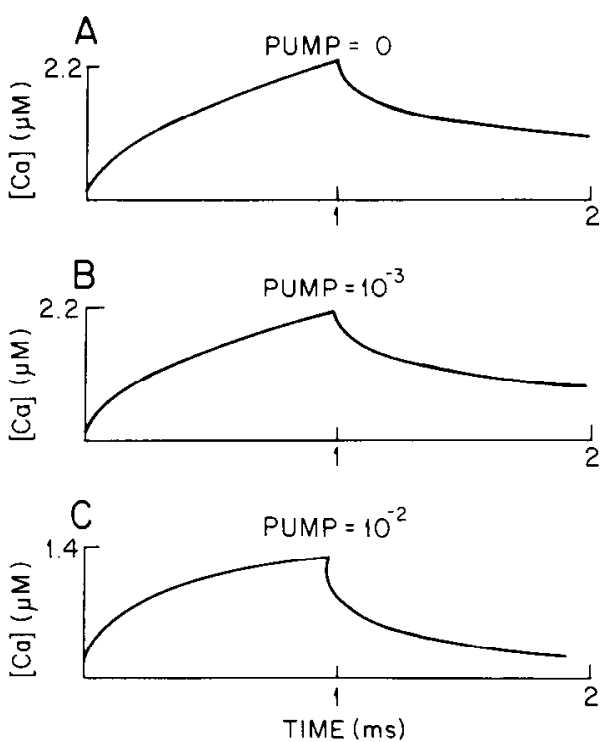

brane available for extrusion becomes determined by the rate of diffusion rather than the pump rate. A 10 -fold increase in the pump rate from $10^{-3}$ to $10^{-2} \mathrm{~cm} / \mathrm{sec}$ causes a drastic change in the time course of the calcium concentration just under the membrane but has little effect on the concentration gradient in the cell. The diffusion gradient, in turn, effectively determined the rate of efflux so that, after a few milliseconds, the efflux was rather insensitive to the pump rate constant.

That the diffusion process limits the pumping rate can be seen perhaps more directly by the following simulations. The same $1-\mu \mathrm{m}$ cell was given an initial uniform concentration of $1 \mathrm{mM}$, and the early time course of efflux following the turning on of a linear pump was plotted in Figure 4 along with the spatial distribution at 100 msec. The concentration gradients at this time are almost the same for the two pump rates $\left(10^{-3}\right.$ and $\left.10^{-2}\right)$.

Transmitter release kinetics. As noted above, the best available measure of transmitter release is the endplate current. These experimental records from neuromuscular junctions of the frog are characterized by (1) a delay of at least $0.5 \mathrm{msec}$ (at $20^{\circ} \mathrm{C}$ and increasing rapidly with cooling, Katz and Miledi, 1965a), (2) a rapid rise to a peak within another 0.05 msec and (3) a slower exponential decay with a time constant of about $1.0 \mathrm{msec}$ (Takeuchi and Takeuchi, 1959; Fig. 5). For comparison of simulations with such records, we chose a reference value for $\beta$ of 50 and a pump rate of $10^{-3}$. A study of the sensitivity of the overall kinetics of release showed that the major observations were relatively independent of the values chosen.

In response to a 1-msec square pulse injection of calcium into the terminal, the internal calcium concentration rises without a delay, but the fourth power of the calcium concentration (which we take as proportional to transmitter release, and hereafter refer to as our "release function"), of course, shows a delay. However, this delay (a fraction of a millisecond) is always small compared to
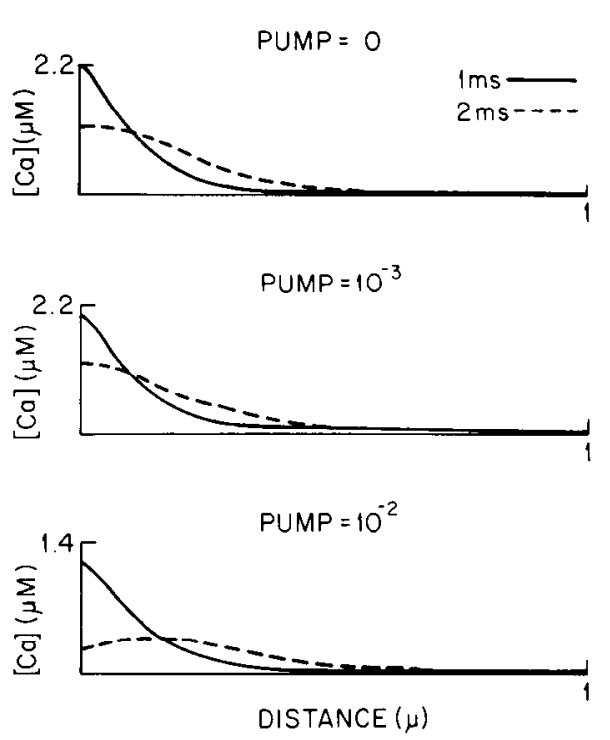

Figure 3. Effect of pump rate on the calcium transient in the model cell for $\beta=50$. As in Figure 2, the left side shows the temporal pattern in the outer $100 \AA$, and the right side shows the spatial gradient at the end of the 1 -msec influx of 1 pmol/cm ${ }^{2}$ and at 5 msec. The pump rates shown are: $A, 0 ; B, 10^{-3} ; C, 10^{-2} \mathrm{~cm} / \mathrm{sec}$. 

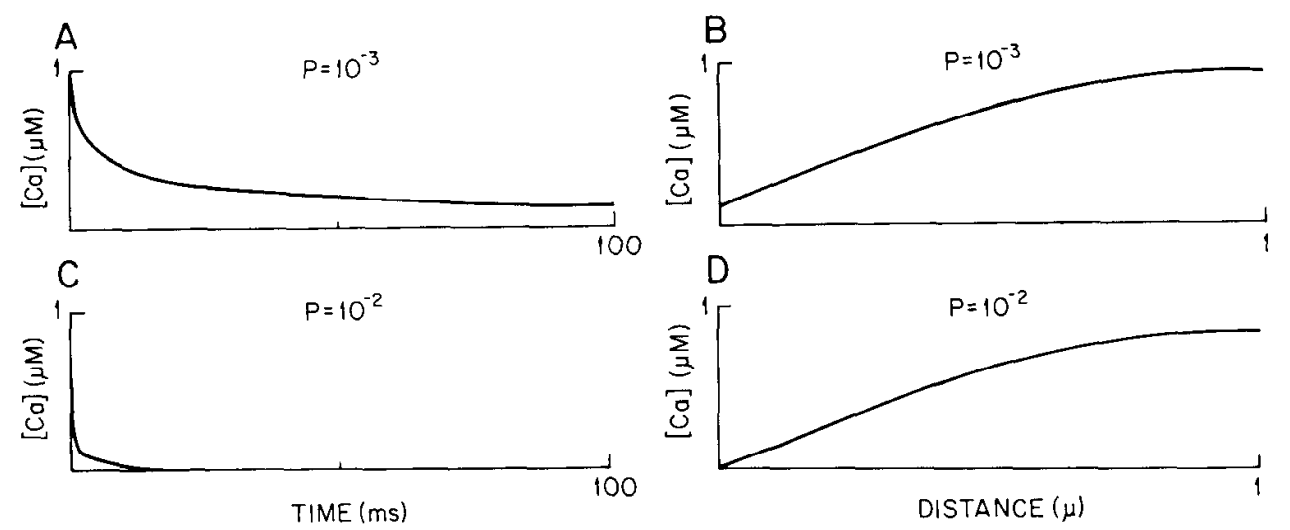

Figure 4. Effect of pump rate on the removal of calcium from a model cell loaded initially with $1 \mathrm{~mm}$ calcium. In $A$ and $B$ the pump is linear with a rate constant of $10^{-3} \mathrm{~cm} / \mathrm{sec}$, and in $C$ and $D$ the rate is $10^{-2} \mathrm{~cm} / \mathrm{sec}$. $A$ and $C$ show the decline of calcium in the outer 100 - $\AA$ shell as a function of time. $B$ and $D$ show the calcium concentration in the model cell as a function of the distance from the surface membrane at $100 \mathrm{msec} . \beta=600$.

the experimentally observed synaptic delay. The rate of fall in submembrane calcium concentration at the end of such a square pulse influx is greater than the rate of rise; the release function, of course, falls even faster. Thus, the rounded peak and relatively slow decay of the endplate current are not fitted with this release function. The substitution of a calcium current of more realistic shape (Llinas et al., 1981a) does not alleviate these two problems.

Clearly, some other mechanism is necessary to account for synaptic delay. Presumably, calcium participates in some reaction to produce release of transmitter, and the additional synaptic delay probably occurs at this stage. Under "Discussion" we will put some constraints on the characteristics such a mechanism must have.

Facilitation. The experimental observations which we would like to reproduce are represented by the data of Mallart and Martin (1967) shown in Figure 6, a semilog plot of the time course of the decay of facilitation. Our first simulations of facilitation used the same parameter values noted above, chosen to match the characteristics of transmitter release. A series of two-pulse simulations were run using a square pulse of current to inject $1 \mathrm{pmol} /$ $\mathrm{cm}^{2}$ of calcium. Figure 6 shows the calcium concentration $(A)$ and the release function $(B)$ from the region $100 \AA$ interior to the surface membrane for a series of twopulse experiments. The initial stimulus results in a release function which is substantially complete by $5 \mathrm{msec}$.

The interval between stimuli was varied from 5 to 100 msec. When the second stimulus was delivered a short time after the first, there is a dramatic increase in the amplitude of the release function for the second. As the interval between stimuli increases, the second release function approached the first in amplitude. The effect of the first influx of calcium on the amplitude of the second clearly extends beyond $100 \mathrm{msec}$. Note that the shape of the release function is well conserved, even when the interval between stimuli is only $5 \mathrm{msec}$. Other simulations showed that the shape of the release function is not dramatically affected by the shape of the calcium influx.

These simulations show a surprisingly good fit to the
Mallart and Martin (1967) data from 5 to $100 \mathrm{msec}$. Although their data for the decay of facilitation was characterized in terms of time constants, it is not appropriate so to describe the results of diffusion and pump processes. For interpulse intervals of less than $5 \mathrm{msec}$ the model predicts an upper limit of facilitation of 15 as the interval is reduced to zero, with a "time constant" of decay less than 2 msec. Both Katz and Miledi (1968) and Barrett and Stevens (1972) showed that the release probability function had a similar rapidly declining early phase with a time constant on the order of 1 msec.

The effect of variations in model parameters on the time course of facilitation has also been investigated. In general, a reduction of the rate of diffusion (increase in $\beta)$ produces a proportional decrease in facilitation at 100 msec but has less effect at shorter times. Similarly, increasing the pump rate reduces late facilitation more than for short times.

\section{Discussion}

'This work shows the very important role of diffusion and pumping of calcium in determining the amplitude and time course of transmitter release and facilitation at a synapse and further defines in considerable detail how "residual calcium" might cause facilitation. The binding of calcium to cytoplasmic sites so reduces diffusion that many tens of milliseconds are required to achieve a uniform distribution of calcium even within a nerve terminal as small as $1 \mu \mathrm{m}$ in diameter. A brief influx produces large local calcium concentration increases just under the cell membrane. Confinement of the calcium load to the region near the membrane enhances the ability of the pump to discharge the calcium and restore a very low cytoplasmic concentration. Thus, the pump also plays an important role in the dynamics of transmitter release.

This slowing of diffusion (by binding of calcium to cytoplasmic elements) has important consequences for any intracellular calcium-dependent process. It means that diffusion cannot be ignored if the time course of the process studied is on the order of milliseconds. For ex- 
ample, the marked decay of inward calcium (but not barium) currents in neurons during maintained depolarization has been ascribed to the calcium-dependent inactivation of the channels. Perhaps, a simpler explanation would be that the influx of calcium significantly alters $E_{\mathrm{Ca}}$ during the voltage clamp pulse. Such an explanation has, in fact, been offered several times (Gorman and Thomas, 1980; Hagiwara and Byerly, 1981). If that were true, a measure of the reversal potential after a long pulse might be expected to demonstrate the reduction. Unfortunately, difficulties measuring the reversal potential and departures of the calcium current from a linear dependence on $E_{\mathrm{Ca}}$ (Hagiwara and Byerly, 1981) make such a test impractical.

There is reason to believe that the actual value of the diffusion coefficient in axoplasm is overestimated (as outlined under "Model") because measurements were
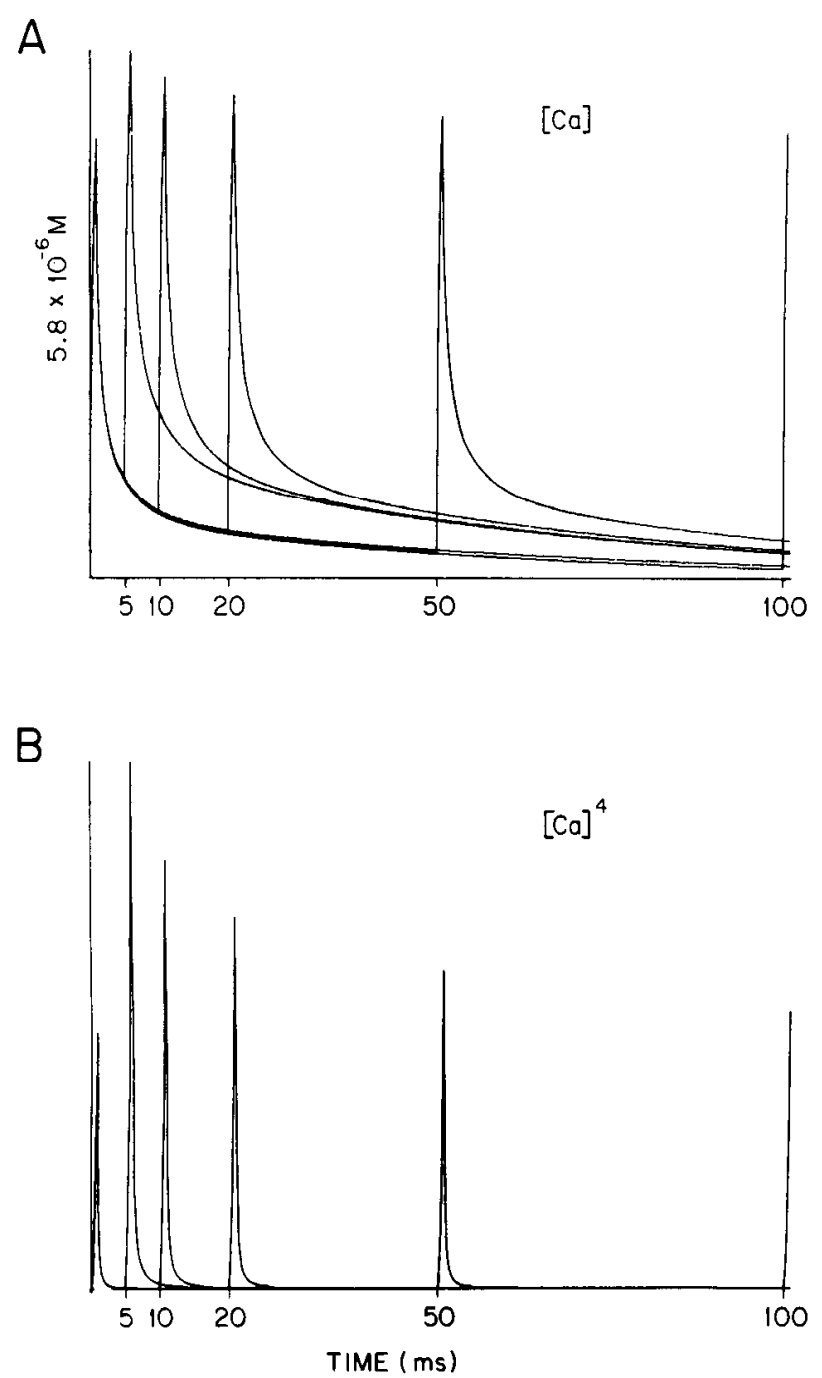

Figure 5. Intracellular calcium transients in response to pairs of brief influxes, between which the time interval was varied. The value of $\beta$ was 50 , and the pump rate was $10^{-3}$. In $A$, the calcium concentration transient in the outer shell (just under the surface membrane) is shown for the conditioning influx at time 0 , and test stimuli at 5, 10, 20,50, and 100 msec. In $B$, the same data are plotted for the fourth power of these calcium transients. Note the basic preservation of the shape of the calcium transient, even when the interpulse interval is $5 \mathrm{msec}$.

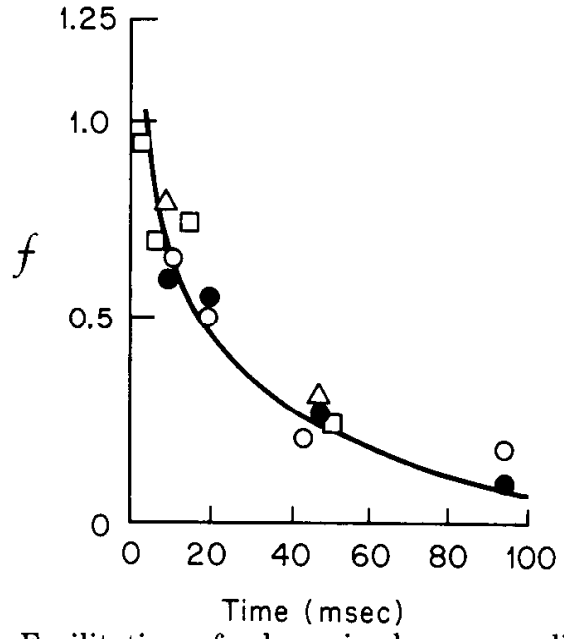

Figure 6. Facilitation of release is shown as a line for the envelope of the peaks of the fourth power of calcium transients from Figure 5. The points are taken from data on the frog neuromuscular junction by Mallart and Martin (1967).

carried out under conditions of high calcium concentrations. Nevertheless, even these measured values are small enough to give rise to the marked spatial and temporal distributions calculated above.

Our model. The model analyzed in the current work differs in a fundamental way from previous efforts (see comparisons below). It consists of the machinery known to exist in the nerve terminal for the influx of calcium, its binding and diffusion within the cell, the proposed relationship between calcium and transmitter release, and, finally, the extrusion of calcium from the terminal, but it includes no components for postsynaptic events.

The model does not include the contribution of mitochondria or smooth endoplasmic reticulum, because they are typically located further than a few hundred angstroms from the release sites and, thus, would have a negligible effect in the time scale of the events we have considered in this paper. Such organelles and their contribution to the maintenance of intracellular calcium levels probably cannot be neglected for such processes as potentiation which occur over a much longer time scale. Contributions made by various intracellular organelles are also very much influenced by slow calcium diffusion. While mitochondria and smooth endoplasmic reticulum are of unquestionable value to the cell in long-term maintenance of the internal calcium levels (Blaustein, 1976; Blaustein et al., 1980), they cannot exert any effects near the membrane on a millisecond to millisecond basis if (1) they are more than a few hundred angstroms away from the external surface membrane and (2) the effects they exert on their own local microenvironments are, because of diffusion, propagated slowly through the rest of the cell.

The present model must be considered in the framework of larger models in which the physiology of some process is seen to depend upon intracellular calcium on a millisecond time scale. It differs from early work of Blaustein and Hodgkin (1969) and Baker et al. (1971) in that it addresses calcium transients as a function of time for arbitrary influxes. It differs from some later models (Andresen et al, 1979; Gorman and Thomas, 1980; Smith 
and Zucker, 1980) in detailing the characteristics of spatial gradients.

Pump rates. The results of our simulations raise the question of accuracy of published values of pump rate constants obtained from experimental measurements of calcium extrusion. The calculated value of the rate constant of a pump is directly proportional to the value of calcium concentration assumed to exist just under the membrane. The simulations of Figure 4 show that as one increases the pump rate, the rate of calcium efflux becomes limited by the rate of diffusion rather than the pump rate.

A second major problem is that the measurement of calcium efflux has very poor temporal resolution because pooled samples are collected over seconds to minutes. The value derived for the maximum flux may be very much underestimated. If at some time a high internal calcium concentration were established and then the pump was turned on, the calcium concentration near the cell membrane may be rapidly reduced by a powerful pump, inducing a gradient, with the concentration higher near the center of the cell and low near the pump at the perimeter, due to slow axial diffusion. But the initial rate measured probably will be in error both because the amount of calcium extruded in the millisecond time scale is still small and because of the finite time required to change solutions. The rate measured at longer times will likely be in error because a reversal of the calcium gradient is achieved within milliseconds, with the lowest calcium concentration near the surface membrane, and further reductions in that pool due to the calcium pump become diffusion limited.

Thus, it appears likely that the magnitude of the published values for pump rates are underestimated in cases where there is significant cytoplasmic binding of calcium.

The saturable nature of calcium efflux measured by Blaustein (1976) must reflect a characteristic of the pump itself, rather than a consequence of diffusion. As the level of cytoplasmic calcium is increased and saturation of binding sites occurs, the effective rate of diffusion will increase. Furthermore, if the internal calcium concentration is raised while the external concentration is maintained, the gradient against which the pump works is decreased. These two effects, if anything, would make the pump more effective in high internal calcium. That it is not more effective would indicate that the saturation observed is an intrinsic property of the pump.

Other facilitation models. Previously two mechanisms have been proposed but have failed to account for facilitation. Broadening of the second nerve terminal spike might result in increases in calcium influx and, therefore, transmitter release. However, Katz and Miledi (1968) were able to produce facilitation at the frog neuromuscular junction when action potentials in the nerve terminal were blocked with tetrodotoxin and release was stimulated by identical electrical depolarizations. Likewise, this hypothesis was not consistent with the observations of uniformity of the nerve terminal potential at the crayfish neuromuscular junction (Zucker and LaraEstrella, 1979) and the squid giant synapse (Charlton and Bittner, 1978). Furthermore, the idea of successive calcium currents increasing, rather than inactivating, is contrary to the findings in the many preparations in which the calcium current has been studied.

The other proposal to account for facilitation has been the residual calcium hypothesis. Although there is no direct evidence for this idea either, Charlton et al. (1982) describe a stepwise increase of the arsenazo III signal in the presynaptic terminal of the squid giant synapse in response to a train of stimuli. This stepwise increase in total intracellular calcium is consistent with both phasic release and facilitation as the calcium load is redistributed toward the center of the terminal (Zucker and Stockbridge, 1983).

Rather than considering component mechanisms, many previously proposed "models" for facilitation have simply employed mathematical functions to describe the rise and fall of the envelope of junction potential amplitudes in response to a train. Few have attempted to include the constraints of phasic release in conjunction with facilitation.

The plausibility of the residual calcium hypothesis was promoted by Younkin (1974), who found that facilitation of endplate potentials could be quite well predicted for a complicated train of impulses based on the fourth power of some intermediate to which successive impulses added equal contributions. However, the decay of the intermediate was fit with a function which bears no apparent relationship to any physical process or even Mallart and Martin's (1967) estimates of decay time constants. Balnave and Gage (1974, 1977) have proposed, in a less quantitative way, a kinetic model for the release mechanism, but they speculate little on the nature of the individual steps.

Rahamimoff (1974) has presented a qualitative model for phasic release and facilitation in which a calcium load binds four ions per site in a random manner. A fully loaded site then results in the release of one quantum of transmitter. The model as presented contained no description of the ultimate fate of internal calcium-no diffusion and no efflux.

Parnas and Segel (1980) have similarly modeled facilitation, but not phasic release, by a scheme in which the prime determinant is the nature of efflux and in which diffusion plays no part.

Llinas et al. (1981b) provide an analysis of phasic transmitter release, but not facilitation, at the squid synapse based on their voltage clamp data of the calcium current in the presynaptic fiber. The model specifically discounts the contribution of diffusion to the time course of transmitter release based on a diffusion constant for calcium of $10^{-6} \mathrm{~cm}^{2} / \mathrm{sec}$. The current work illustrates that use of values one or two orders of magnitude lower, a more realistic estimate of the diffusion constant for calcium, means that diffusion cannot be dismissed as an important contributor to the time course of transmitter release.

Summary. Although it is a much oversimplified representation of the nerve terminal, this model shows that diffusion plays an important role in determining the time course of phasic release and facilitation. It predicts, for reasonable values of the effective diffusion constant and the pump rate, transmitter release that begins after a delay, rises rapidly to a peak, and then decays more slowly, reaching half the peak value in about another 
millisecond. Some fraction of the delay in the onset of transmitter release may be due to diffusion either radially or laterally, but the steep temperature dependence argues against diffusion being a major determinant in synaptic delay.

In addition to having transmitter release turn off within a few milliseconds, this model predicts well the amplitude and time course of facilitation of transmitter release to later stimuli, while preserving the shape of the release function. Furthermore, it predicts a decay of facilitation which, while not exponential, would be as adequately fit with a time constant of $35 \mathrm{msec}$ (in the range of 10 to $100 \mathrm{msec}$ ) as are experimental observations. The gradual departure from an exponential suggests a component with a longer time constant as well.

\section{References}

Andresen, M. C., A. M. Brown, and S. Yasui (1979) The role of diffusion in the photoresponse of an extraretinal photoreceptor of Aplysia. J. Physiol. (Lond.) 287: 283-301.

Andreu, R., and E. F. Barrett (1980) Calcium dependence of evoked transmitter release at very low quantal contents at the frog neuromuscular junction. J Physiol. (Lond.) 308: 79 97.

Baker, P. F. (1972) Transport and metabolism of calcium ions in nerve. Prog. Biophys. Molec. Biol. 24: 177-223.

Baker, P. F. (1976) Regulation of intracellular calcium and magnesium in squid axons. Fed. Proc. 35: 2589-2595.

Baker, P. F., A. L. Hodgkin, and E. B. Ridgeway (1970) Two phases of calcium entry during the action potential in giant axons of Ioligo. J. Physiol. (Lond.) 208: 80P.

Baker, P. F., A. L. Hodgkin, and E. B. Ridgeway (1971) Depolarization and calcium entry in squid giant axons. J. Physiol. (Lond.) 218: 709-755.

Balnave, R. J., and P. W. Gage (1974) On facilitation of transmitter release at the toad neuromuscular junction. J. Phyșiol. (Lond.) 239: 657-675.

Balnave, R. J., and P. W. Gage (1977) Facilitation of transmitter secretion from toad motor nerve terminals during brief trains of action potentials. J. Physiol. (Lond.) 266: 435-451.

Barrett, E. F., and C. F. Stevens (1972) The kinetics of transmitter release at the frog neuromuscular junction. J. Physiol. (Lond.) 227: 691-708.

Blaustein, M. P. (1976) The ins and outs of calcium transport in squid axons: Internal and external ion activation of calcium efflux. Fed. Proc. 35: 2574-2578.

Blaustein, M. P., and A. L. Hodgkin (1969) The effect of cyanide on the efflux of calcium from squid axons. J. Physiol. (Lond.) 200: 497-527.

Blaustein, M. P., R. W. Ratzlaff, and E. S. Schweitzer (1980) Control of intracellular calcium in presynaptic nerve terminals. Fed. Proc. 39: 2790-2795.

Charlton, M. P., and G. D. Bittner (1978) Presynaptic potentials and facilitation of transmitter release in the squid giant synapse. J. Gen. Physiol. 72: 487-511.

Charlton, M. P., S. J. Smith, and R. S. Zucker (1982) Role of presynaptic calcium ions and channels in synaptic facilitation and depression at the squid giant synapse. J. Physiol. (Lond.) 323: 173-193.

Crank, J. (1956) The Mathematics of Diffusion, Clarendon Press, Oxford.

DiPolo, R., and L. Beaugé (1979) Physiological role of ATPdriven calcium pump in squid axon. Nature (Lond.) 278: 271273.

Dodge, F. A., Jr., and R. Rahamimoff (1967) Cooperative action of calcium ions in transmission release at the neuromuscular junction. J. Physiol. (Lond.) 193: 419-432.

Gorman, A. L. F., and M. V. Thomas (1980) Intracellular calcium accumulation during depolarization in a molluscan neurone. J. Physiol. (Lond.) 308: 259-285.

Hagiwara, S., and L. Byerly (1981) Calcium channel. Annu. Rev. Neurosci. 4: 69-125.

Hodgkin, A. L., and R. D. Keynes (1957) Movements of labelled calcium in squid giant axon. J. Physiol. (Lond.) 138: 253281.

Katz, B., and R. Miledi (1965a) The effect of temperature on the synaptic delay at the neuromuscular junction. J. Physiol. (Lond.) 181: 656-670.

Katz, B., and R. Miledi (1965b) The effect of calcium on acetylcholine release from motor nerve terminals. Proc. $R$. Soc. Lond. Biol. 161: 491.

Katz, B., and R. Miledi (1967a) The timing of calcium action during neuromuscular transmission. J. Physiol. (Lond.) 189: 535-544.

Katz, B., and R. Miledi (1967b) The release of acetylcholine from nerve endings by graded electrical pulses. Proc. R. Soc. Lond. Biol. 167: 23-38.

Katz, B., and R. Miledi (1968) The role of calcium in neuromuscular facilitation. J. Physiol. (Lond.) 195: 481-492.

Llinas, R., I. Z. Steinberg, and K. Walton (1981a) Presynaptic calcium currents in squid giant synapse. Biophys. J. 33: 289322.

Llinas, R., I. Z. Steinberg, and K. Walton (1981b) Relationship between presynaptic calcium current and postsynaptic potential in squid giant synapse. Biophys. J. 33: 323-352.

Magleby, K. L., and C. F. Stevens (1972) The effect of voltage on the time course of end-plate currents. J. Physiol. (Lond.) 223: 151-171.

Mallart, A., and A. R. Martin (1967) An analysis of facilitation of transmitter release at the neuromuscular junction of the frog. J. Physiol. (Lond.) 193: 679-694.

Miledi, R. (1973) Transmitter release induced by injection of calcium ions into nerve terminals. Proc. R. Soc. Lond. Biol. 183: 421-425.

Mullins, L. J. (1976) Steady-state calcium fluxes: Membrane versus mitochondrial control of ionized calcium in axoplasm. Fed. Proc. 35: 2583-2588.

Parnas, H., and L. A. Segel (1980) A theoretical explanation for some effects of calcium on the facilitation of neurotransmitter release. J. Theor. Biol. 84: 3-29.

Rahamimoff, R. (1974) Modulation of transmitter release at the neuromuscular junction. In The Neurosciences, Third Study Program, F. O. Schmidt and F. G. Worden, eds., pp. 943-952, MIT Press, Cambridge, MA.

Rahamimoff, R., S. D. Erulkar, A. Lev-Tov, and H. Meiri (1978a) Intracellular and extracellular calcium ions in transmitter release at the neuromuscular synapse. Ann. N. Y. Acad. Sci. 307: 583-598.

Rahamimoff, R., H. Meiri, S. D. Erulkar, and Y. Barenholz (1978b) Changes in transmitter release induced by ion-containing liposomes. Proc. Natl. Acad. Sci. U. S. A. 75: 52145216.

Smith, S. J., and R. S. Zucker (1980) Aequorin response facilitation and intracellular calcium accumulation in molluscan neurones. J. Physiol. (Lond.) 300: 167-196.

Takeuchi, A., and N. Takeuchi (1959) Active phase of frog's endplate potential. J. Neurophysiol. 22: 395-411.

Younkin, S. G. (1974) An analysis of the role of calcium on facilitation at the frog neuromuscular junction. J. Physiol. (Lond.) 237: 1-14.

Zucker, R. S., and L. O. Lara-Estrella (1979) Is synaptic facilitation caused by presynaptic spike broadening? Nature (Lond.) 278:57-59.

Zucker, R. S., and N. Stockbridge (1983) Presynaptic calcium diffusion and the time courses of transmitter release and synaptic facilitation at the squid giant synapse. J. Neurosci. 3: 1263-1269. 